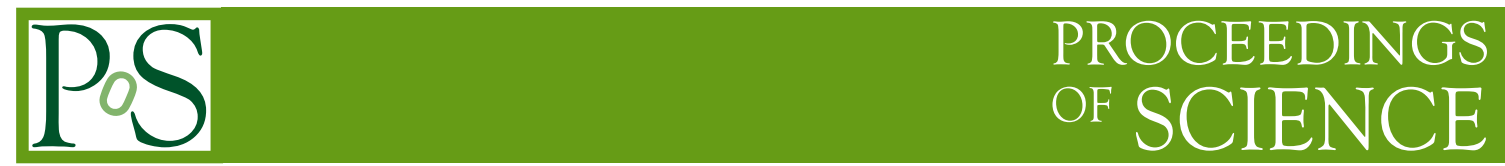

\title{
Topical summary of experimental results
}

\author{
Kerstin Borras \\ Deutsches Elektronen-Synchrotron \\ DESY, Notkestrasse 85, 22603 Hamburg, Germany \\ E-mail: borras@mail.desy.de
}

The experimental results presented during the international "Diffraction 2006" workshop span an incredibly large variety of physics aspects. This paper introduces into the full scope of the presentations by briefly summarizing the reported physics topics, putting individual results into perspective and connecting them as much as possible.

Diffraction 06, International Workshop on Diffraction in High-Energy Physics

September 5-10, 2006

Adamantas, Milos island, Greece 


\section{Introduction}

The experimental results, presented during the six days of the Diffraction 2006 workshop in Greece, span an incredibly large variety of physics topics. In total we heard 21 talks covering 11 hours. Compared to the previous conferences now the experimental reports are in balance with the theoretical talks and a firm basis for fruitful interactions between experimentalists and theorists is built. This summary puts the individual results into perspective and connects them as much as possible. In the following no special explanations are given in detail, since those and the relevant references can be found in the excellently prepared proceedings of all talks.

The red line followed in this paper starts with parton densities, their measurements at HERA, their uncertainties and tests performed in measurements at Tevatron. The spin structure of the nucleon, the gluon polarization being one part of it, will be briefly mentioned. Exclusive production processes for vector mesons or DVCS (Deeply Virtual Compton Scattering) give decisive boundary condition for the gluon distribution and the parton evolution. These processes provide access to the so-called generalized parton distribution functions.

The next knot in the red line is given by the results reported on diffraction. First the inclusive diffraction measurements at HERA are discussed, followed by the studies of the diffractive production of special hadronic final states like dijets and charm. Closely connected to these topics is the leading baryon production.

Going over from the results in $e p$ collisions at HERA to the hadron colliders, first to the $\bar{p} p$ collisions at the Tevatron and then to RHIC, leads to the preparations and expectations for the $p p$ collisions at the LHC.

At the end of the red line the future prospects for HERA and the Tevatron appear and the present plan for the first LHC running is briefly outlined.

\section{Inclusive Measurements, Parton Densities and their Uncertainties}

The workshop started with an overview on the recent results from inclusive measurements at HERA (J.Kretschmar). Comparing the results derived from the first $30 \mathrm{nb}^{-1}$ from 1992, accessing a precision of roughly $20 \%$, an incredible accuracy is now achievable with the new data. With a statistics about 10 times larger the analysis of the 1997 data decreases the uncertainty down to $2 \%-3 \%$. The similar amount of $e^{+} p$ and $e^{-} p$ in connection with varying polarization opens the window to precision measurements and their comparison to prediction of the Standard Model. In order to further increase the statistics and the significance the H1 and ZEUS Collaborations have established common working groups combining relevant results. One extraordinary result of such an effort is the determination of the polarization asymmetry $A^{ \pm}$. With this analysis parity violation is observed for the first time in the $\gamma-Z$ interference with $\mathrm{NC} e^{ \pm}$data at radii down to $10^{-18} \mathrm{~m}$.

Another area of a common working group is the extraction of the parton distribution functions (PDF's). By including the jet production data the ZEUS Collaboration could well decrease the uncertainty of the gluon distribution especially at high $x$ and separate the gluon evolution from the $\alpha_{s}$ measurement. The combination of all HERA data, including the semi-inclusive data on jets and heavy quarks, may lead to a further step towards higher precision. 


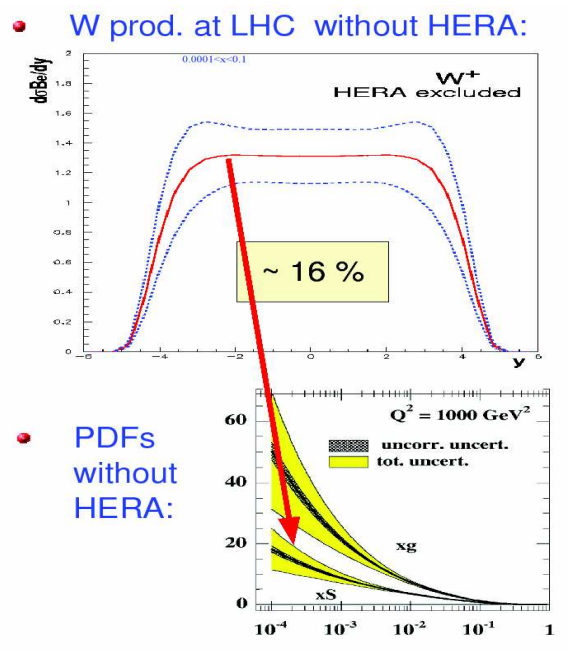

- W prod. at LHC including HERA
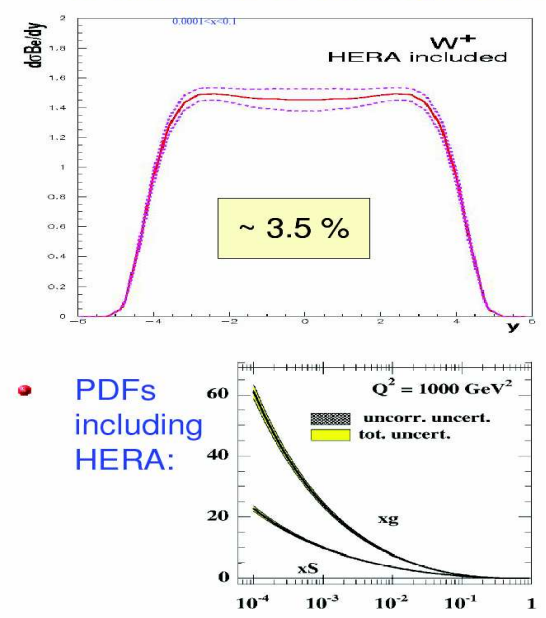

Figure 1: Uncertainty in the measurement of $W$-production at the LHC without using HERA data(left) and with including HERA data(right).

As pointed out (J.Kretzschmar) the central production of heavy particles at the LHC probes the parton distribution functions in the HERA domain. The relevance of a precise determination of the parton densities can be demonstrated with the example of $W$-production at the LHC. Neglecting the input from HERA would lead to an uncertainty of about $16 \%$ as seen on the left side of figure 1 . Including the accurate data from HERA decreases the uncertainty down to approximately $3 \%$.

The uncertainty of the parton distribution functions are vivid analysis topics at the hadron collider Tevatron. The impact of the present uncertainties on specific measurements were demonstrated and different possibilities with their power on constraining the parton distribution functions were discussed in two presentations (A.Robson, V.O'Dell). Uncertainties in the measurements result from uncertainties in the PDF's via acceptance calculations or background estimates. Theoretical calculated cross sections are directly dependent on the PDF's.

One prominent analysis example is the inclusive jet cross section, which provides information on the gluon distribution at high values of $x$. Measurements like the $Z$ boson rapidity, forward produced $W$-bosons or $Z$-boson production combined with $b$-quarks are promising channels. The progress achieved so-far and possibly reachable in future can be well demonstrated with the example of the $W$ charge asymmetry, which is sensitive to the $d / u$ ratio. In 1998 the CDF measurements lead to a $30 \%$ increase of the d-quark distribution at a $Q^{2}$ of about $20 \mathrm{GeV}^{2}$. Both the CDF and the D0 Collaboration have increased the accuracy of this measurement with the Run II data. Now the uncertainty originating from the PDF uncertainties is larger than the experimental uncertainties. This fact becomes even more pronounced in the projected statistical uncertainty for the data collected with envisaged $400 \mathrm{pb}^{-1}$ as displayed in figure 2 . As the statistics for the Tevatron data is continously increasing here promising outlooks are in view. 


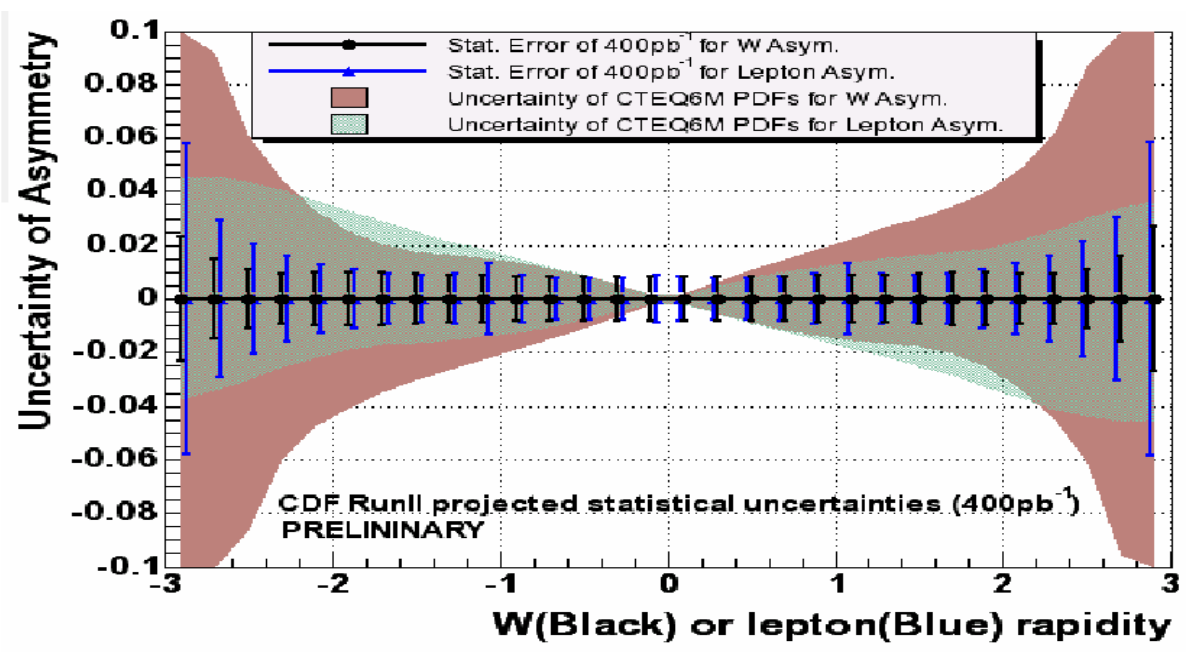

Figure 2: Uncertainty in the measurement of charge asymmetry in $W$ and Lepton- production at the Tevatron projected for $400 \mathrm{pb}^{-1}$.

\section{Polarized Parton Densities}

The aspect of spin effects was introduced to the workshop by A.Krisch, who reviewed the past, present and the future of spin experiments with high energetic proton beams [1]. The still unexplained large transversal spin effects found in the past are the seed for a future experiment possibly transfered from Protvino in Russia to J-Park in Japan.

Several running experiments are presently studying the spin composition of nucleons, assigning fractions of the nucleons helicity to quarks, gluons and the orbital angular momentum: $1 / 2=1 / 2 \Delta \Sigma+\Delta G+\Delta L$. The different kinematic regions accessible to the individual experiments is clearly seen in a drawing copied from L.Jenkovszky's talk as displayed in figure 3.

Several new results from the COMPASS experiment concerning for example the longitudinal helicity asymmetry $A_{1}^{d}$ and the derived spin-dependent structure function $g_{1}^{d}$ were presented by K.Kurek. They reveal agreement with the previous results from other experiments but with a much improved accuracy due to the considerably increased statistics.

A special interesting subject is the gluon polarization $\Delta G / G$. Since the derivation of this quantity from QCD-fits allows both negative and positive values for $\Delta G$ within the present uncertainty, it is preferable to pursue direct measurements of this observable. COMPASS achieved new preliminary results from studies of the open charm channel and of the production of high $p_{T}$ hadrons. Latter ones were analyzed separately for $Q^{2}$ smaller and larger than $1 \mathrm{GeV}^{2}$. Putting all measurements from the different experiments together in one plot visualizes that the value for $\Delta G$ is still allowed to lie between 0.4 and -0.6 with large uncertainties. While $\Delta G=0.4$ is not excluded and with this opening the possibility for a scenario where $\Delta L$ is small, a value of $\Delta G$ close to zero would indicate the important role of the angular orbital momentum in the nucleon spin decomposition as described in the framework of the parton model and QCD. 

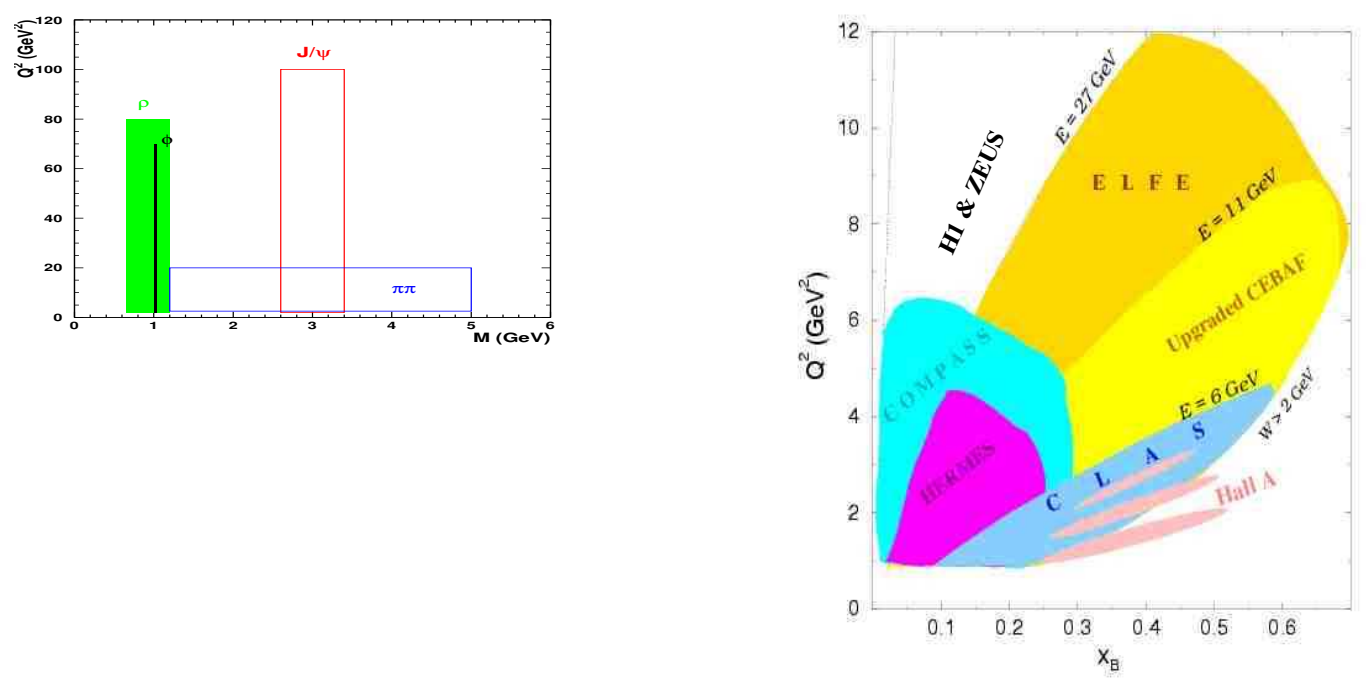

Figure 3: Examples for vector meson and DVCS analyses (left) and kinematic region accessible to the various experiments.

The first (model-dependent) constraint on the total angular momenta of the $\mathrm{u}$ - and d- quarks $\left(J_{u}, J_{d}\right)$ has been reported by HERMES (R.Fabbri). In order to unfold the relation of $J_{u}$ and $J_{d}$ a model with a certain set of Generalized Parton Distribution functions (GPD's) has been used to interpret the Deeply Virtual Compton Scattering (DVCS) data exploiting the sensitivity of the transverse target spin asymmetry to $J_{q}$.

This result leads directly over to the next knot in the red line of this summary, the measurements of exclusive processes and their interpretation in which both DVCS and GPD's play a crucial role.

\section{Exclusive Processes}

Exclusive processes have the advantage of a clean experimental signature. In case of DVCS or high- $t$ photon production the final state omits even the step of hadronisation: the soft part of the production of the vector meson and its subsequent decay. With the five reports (N.Berger, M.Beckingham, A.Borissov, R.Fabbri, D.Szuba) a nice overview was given on this subject and new results were presented.

The opportunity to set different scales in the exclusive production of vector mesons opens the unique possibility to study diffractive exchanges in the soft or in the hard regime and also the transition region between these two distinct regimes in various aspects. Hard scales needed for perturbative QCD (pQCD) calculations can be set for example by the choice of $Q^{2}$, the virtuality of the photon, $W$, the center of mass energy of the $\gamma p$ system, $M_{V M}$, the mass of the vector meson, or by $t$, the squared four-momentum transfer at the proton vertex. Selecting events with a high value of $t$ means predominantly selecting events with the proton been dissociated ( $p$-diss) due to the high momentum transfer. These processes are shown in the two middle pictures of figure 4. For lower values of $t$ the proton stays usually intact as depicted in the left picture of figure 4 . 

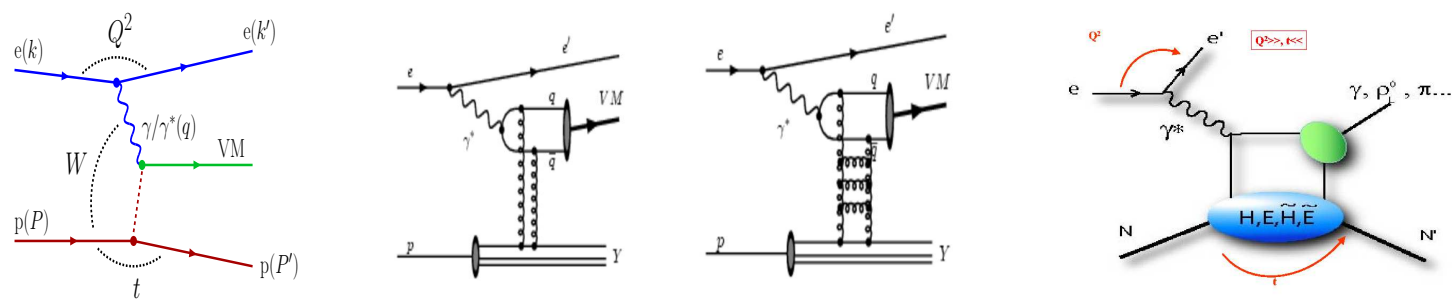

Figure 4: Models for vector meson production in the framework of Regge Phenomenology (left), two-gluon or gluon ladder exchanges in Color Dipole models (middle) and GPD's (right).

The left side of figure 3 displays the various vector mesons studied in the different experiments. Also exclusive di-pion events are analyzed in the marked mass range (D.Szuba). On the right side of the figure the kinematical regions accessible for the different experiments are compared (L.Jenkovszky).

In figure 4 three different approaches to describe exclusive vector meson production and DVCS are drawn. Soft processes like the exclusive photoproduction (PHP) with $Q^{2} \approx 0$ of e.g. $\rho$ or $\omega$ reveal a weak dependence on $W$ with a power law behavior: $W^{\delta}$. These types of processes can be well described within the Regge-approach displayed on the left side of figure 4. By analyzing simultaneously the $W$ dependence in different bins of $t: W^{4\left(\alpha_{I P}(t)-1\right)}$ an effective Pomeron trajectory $\alpha_{I P}(t)=\alpha_{0}+\alpha^{\prime} \cdot t$ can be extracted from one single experiment. The H1 Collaboration measured it recently for exclusive $\rho$ production with the new 2005 data: $\alpha_{I P}(t)=1.093 \pm 0.003_{-0.007}^{+0.008}+$ $0.116 \pm 0.027_{-0.046}^{+0.036} \cdot t$. These values are in good agreement with the previously published values of the ZEUS and the OMEGA Collaboration. They again reveal the remarkable feature that $\alpha^{\prime}$ is significantly smaller than the commonly cited value for the so-called soft pomeron of $0.25 \mathrm{GeV}^{-2}$ as published by Donnachie and Landshoff in 1992.

The $W$ dependence is steepening with increasing values for the power $\delta$ when going from the photoproduction to the DIS regime with its larger values of $Q^{2}$ or to vector mesons with larger masses like the $J / \Psi$. Then the power $\delta$ approaches values predicted by pQCD calculations in the framework of Color Dipole models (two middle pictures in figure 4) or GPD's (right picture of figure 4). It was pointed out by D.Szuba, that the resulting Pomeron trajectory is different for different scales as given by $J / \Psi$ production in PHP, in DIS or with high $t$.

\begin{tabular}{|l|l|l|}
\hline process & $\alpha_{0}$ & $\alpha^{\prime}$ \\
\hline$\gamma p \rightarrow J / \Psi p$ & $1.200 \pm 0.009$ & $0.115 \pm 0.018$ \\
$\gamma * p \rightarrow J / \Psi p$ & $1.20 \pm 0.03$ & $0.07 \pm 0.05$ \\
$\gamma p \rightarrow J / \Psi Y$ & $1.153 \pm 0.048$ & $0.020 \pm 0.014$ \\
\hline
\end{tabular}

Table 1: Pomeron trajectories for $J / \Psi$ in PHP, in DIS or with high $t$.

These results are summarized in table 1 and in figure 5. With those results the postulate of a universal pomeron is put into question and one has to ask for the definition of the appropriate scale, which might depend on a combination of $Q^{2}, M_{V M}^{2}$ and $t$. 


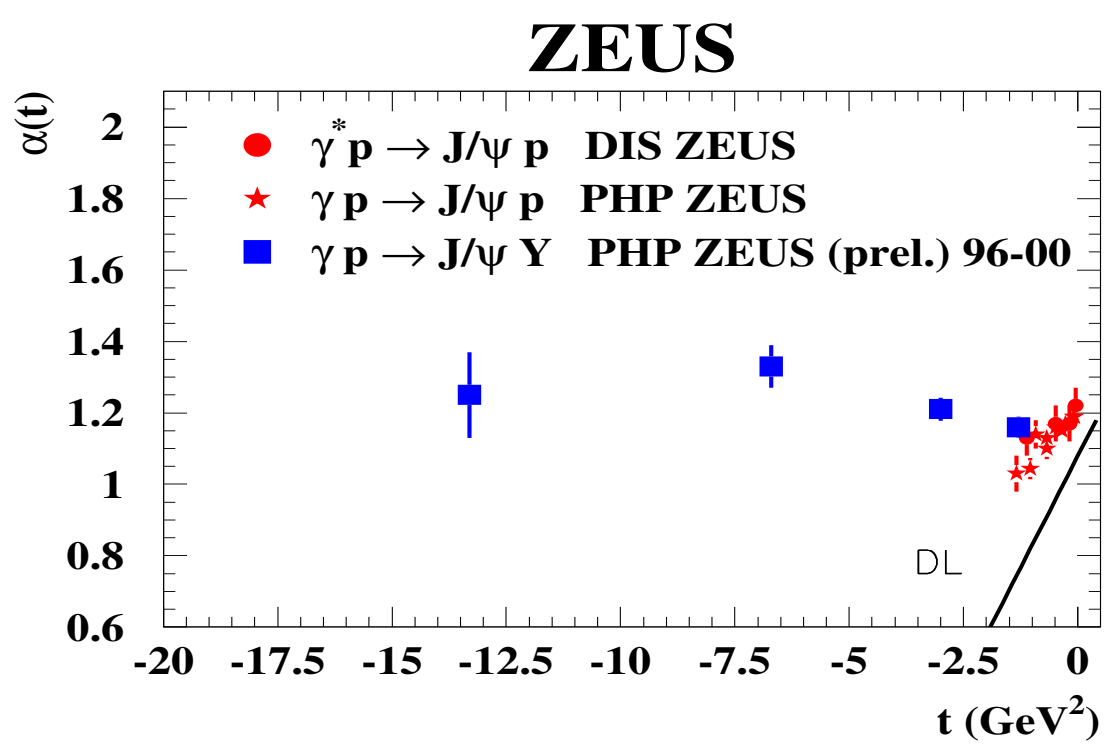

Figure 5: Pomeron trajectories for $J / \Psi$ in PHP, in DIS or with high $t$.

For vector meson production with a hard scale involved, i.e. higher $Q^{2}$ in DIS or the production at high $t$ or vector mesons with larger masses, pQCD calculations using two-gluon or gluon ladder exchanges are possible. Some of the calculations are performed in the framework of a dipole model. Common to the models is the high sensitivity to the gluon density of the proton as pointed out by N.Berger using the example of elastic $J / \Psi$ production in DIS. The $Q^{2}$ dependence reveals large differences when employing different proton parton distribution functions especially at higher values of $Q^{2}$ above $10 \mathrm{GeV}^{2}$. Unfortunately at those values of $Q^{2}$ also the experimental uncertainties are relatively large. The $W$ dependence exhibits an even stronger sensitivity to the gluon distribution over almost the full range of accessible $W$ values. In contrast to the $Q^{2}$ dependence the differences between the predictions from the various proton PDF's are larger and well above the experimental uncertainties. The $t$ dependence and again the $W$ dependence was analyzed for $J / \Psi$ production at high $t$ (D.Szuba) and compared to calculations using DGLAP or BFKL evolution. While the $t$ dependence of the data is reproduced by both, the $W$ dependence reveals disagreement between the DGLAP calculations and the data.

Assuming a flavor independent production mechanism asymptotic SU(4) pQCD predicts the following ratios of vector meson production cross sections: $\rho: \omega: \phi: J / \Psi=9: 1: 2: 8$. HERMES has measured this ratio for $\phi$ and $\rho$ and found beside the known breaking of the flavor independent production at low $Q^{2}$ also a $W$ dependence of this cross section ratio (A.Borissov).

Vector meson production is also a rich testing ground for the Generalized Parton Distribution functions. These are a continuation of the usual parton distribution functions taking into account correlations between the partons in the proton. Besides the usual dependence of the parton distribution functions on $x$ and the scale $Q^{2}$ (here $\mu^{2}$ ) the GPD's also depend on the skewedness, $\xi$, the longitudinal momentum fraction of the proton going into the hard sub-process and also on $t$. For meson production the additional soft process for the hadronisation into final meson state has to be 
taken into account. The production of different mesons project out different GPD's: pseudo-scalar channels for the polarized and longitudinally polarized vector meson channels for the unpolarized GPD's. Impressive examples for the selective determination of the different GPD's by analyzing different meson states were presented by R.Fabbri. One of them is the hard exclusive pion pair production. The measured values for the Legendre moment as a function of $x$ and calculations for flavor non-singlet quark combinations in addition to the dominant singlet exchange in the framework of GPD's, are found to be in fair agreement with the data. The calculations are seen to describe the increased contribution of the non-singlet part with increasing $x$.

The cleanest access to GPD's is provided by the process of DVCS, where a quark is absorbing a virtual photon in DIS and radiating a real photon. This process avoids the additional soft part of meson production. HERMES uses different cross section asymmetries in the azimuthal angle $\phi$ to access the DVCS amplitude through the interference term between the dominant Bethe-Heitler process and DVCS. One prominent analysis result has been mentioned already in the previous section, the first model dependent constraint on the total angular momenta $J_{u}$ and $J_{d}$.

DVCS processes are also analyzed by the two other HERA experiments, H1 and ZEUS, which provide information in a different kinematic region as the HERMES data. New preliminary results from the $\mathrm{H} 1$ experiment using recent HERA II data are in good agreement with the published results obtained by $\mathrm{H} 1$ and ZEUS from data taken during the HERA I running phase (M.Beckingham). Also the related process of diffractive high $t$ photon production has been analyzed by H1. In contrast to DVCS, the latter takes place at low $Q^{2}$ and high $t$. As in the case of vector meson production at high $t$ the data are in reasonable agreement in shape and normalization with calculations from a LLA BFKL approach.

\section{Diffraction and Leading Baryons at HERA}

Incredible progress from the inclusive diffractive DIS front has been reported in two talks by H1 and ZEUS (P.Laycock, B.Löhr). The H1 Collaboration has recently finally published their studies of the 1997 data together with the high $Q^{2}$ data recorded in 99-00. Now also the diffractive parton densities resulting from the DGLAP QCD analysis of the $Q^{2}$ and $\beta$ dependences are finally published. In addition new preliminary results from the analysis of the remaining low $Q^{2}$ HERA I data taken in 99-00 and from first HERA II taken in 2004 were presented. The ZEUS Collaboration managed to apply the three available different selection methods for diffraction, the large rapidity gap selection, the detection of a diffractively scattered proton with a leading proton spectrometer and the $M_{x}$ method to the same data taking period in 99-00 and presented new preliminary results for all selection methods. Especially the ZEUS detector is well suited for detailed comparisons of the three methods, because it offers, due to the additional Forward Plug Calorimeter, the largest kinematic phase space for the application of the $M_{x}$ method. Reasonable agreement is found between the different results from both ZEUS and H1 data within the quoted errors. Few remaining differences have still to be resolved until a consistent picture of inclusive diffraction at HERA will be achieved.

One point of debate was a possible dependence of the Pomeron intercept $\alpha_{I P}(0)$ on $Q^{2}$. The independence of the Pomeron intercept of $Q^{2}$ is a central assumption in the proton vertex factorization, which factorizes the inclusive diffractive DIS cross section into two parts: one depending 

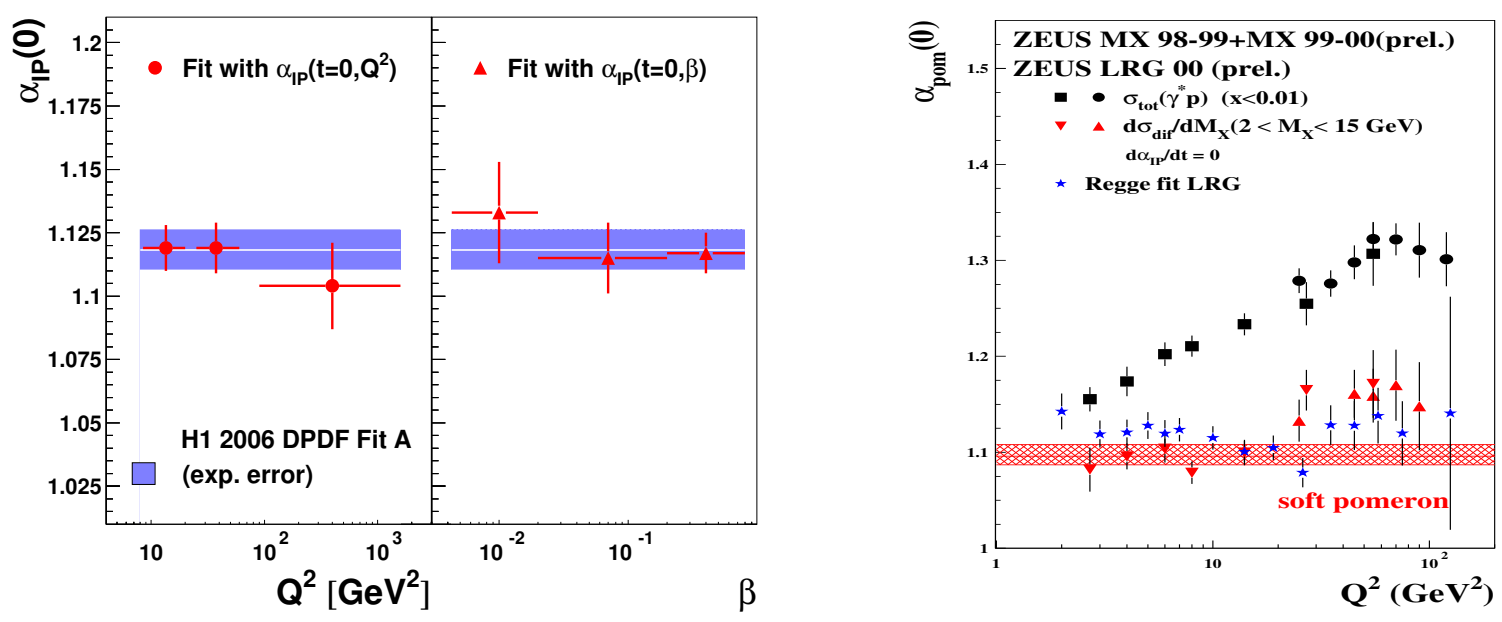

Figure 6: Intercept of the Pomeron as a function of $Q^{2}$ and $\beta$ as measured with the rapidity gap by the H1 Collaboration and with both the rapidity gap and the $M_{X}$ method by the ZEUS Collaboration.

only on quantities at the proton vertex $x_{I P}$ and $t$, where $x_{I P}$ is the longitudinal momentum fraction of the proton entering the hard sub-process. The other part is then only depending on $\beta$ and $Q^{2}$. With this pre-requisite it is possible to analyze first the soft part of the diffractive interaction and parameterize it as a function of $x_{I P}$ and $t$ according to dependences derived from earlier hadronhadron measurements and then to apply a DGLAP CQD fit procedure to the remaining part of the hard parton scattering process. Figure 6 displays the result for the Pomeron intercept from $\mathrm{H} 1$ on the left side as a function of $Q^{2}$ and $\beta$ from the large rapidity gap selection. On the right side the analysis results from ZEUS employing the large rapidity gap selection and the $M_{x}$ method are shown. While both results derived from the large rapidity gap selection are within the errors in agreement with a flat behavior, the results of the $M_{x}$ method resembles a bit the rising dependence on $Q^{2}$ of the inclusive DIS measurement.

In contrast to the usually quoted fraction of $10 \%$ diffraction in inclusive DIS at HERA figure 7 shows a clear decrease of the diffractive contribution to the total cross section going down from approximately $16 \%$ at low $Q^{2}$ to about $2 \%$ at high values of $Q^{2}$. These $2 \%$ are quite close to the usually quoted rate of diffraction of $1 \%$ at the Tevatron at a comparable value of the hard scale.

The analysis of events with specific final states like jets or $D^{*}$ provides the opportunity to test the QCD factorization theorem, which is proven for diffraction in DIS. If the QCD factorization holds, then the measured production cross sections for these events should be in agreement with $\mathrm{pQCD}$ calculations using the diffractive parton densities as derived from inclusive measurements. This is found to be true for diffractive production of dijets and charm in DIS (M.Mozer, I.Korzhavina). Here the dijet data again provide valuable information on the diffractive PDF's, especially for the gluons at high values of $\beta$. Equivalent to the inclusive case, these dijet data can be used in addition in the DGLAP QCD fits and decrease the PDF uncertainties at high $\beta$. It is remarkable, that the use of the dijet data lead to even lower gluon densities at high $\beta$ as derived from the inclusive data alone.

For photoproduction the QCD factorization is not proven, just in contrast. In PHP the pho- 
2006/08/10 17.03

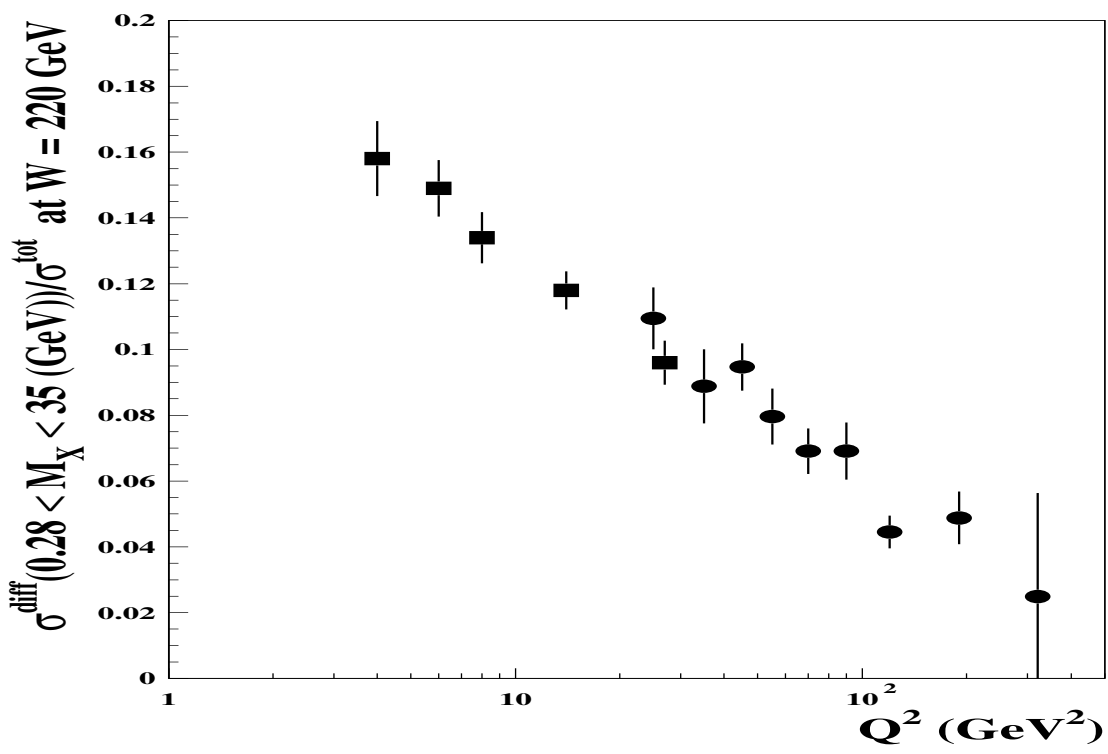

Figure 7: Ratio of the diffractive and the total cross section as a function of $Q^{2}$ measured with the $M_{X}$ method by the ZEUS Collaboration.

ton is quasi-real and acts hadron-like. This means that additional soft rescattering processes as in hadron-hadron scattering can occur, which are assumed to cause a violation of the QCD factorization. Within the low statistics and the large uncertainties of the NLO calculations for the cross sections no significant violation of factorization is observed for diffractive charm production in PHP. But in the dijet data the cross section for both, the direct and the resolved photon interaction, cannot be satisfactorily described with the NLO calculations. It is remarkable, that not only in the resolved case too low cross sections are measured, but also in the direct case, which is in the photon interaction equivalently point-like as in DIS, where factorization was found to hold.

Also in leading baryon production at HERA factorization is studied (L.Rinaldi). Analyzing for example the leading neutron yield as a function of $x_{L}$, the momentum fraction of the proton taken by the leading neutron and as a function of the $p_{T}$ of the neutron, separately for DIS and PHP, reveals absorptive effects symptomatic for factorization breaking. Model calculations in the same framework of rescattering processes as applied for the predictions for dijet production in PHP at HERA or in $\bar{p} p$ collisions at the Tevatron are in agreement with the measurements. Therefore the leading baryon production at HERA helps to adjust model parameters crucial for the calculation of exclusive processes at LHC, one prominent example being the exclusive Higgs production as a discovery channel. Another testing ground for those predictions are the diffractive measurements at the Tevatron, which build the next knot in the red line of this summary of the presented experimental results.

\section{Diffraction at the Tevatron}

The report on the diffractive physics program pursued by the DØ experiment (V.O'Dell) was centered on the operation of the new spectrometers available now in Run II. Forward scattered 

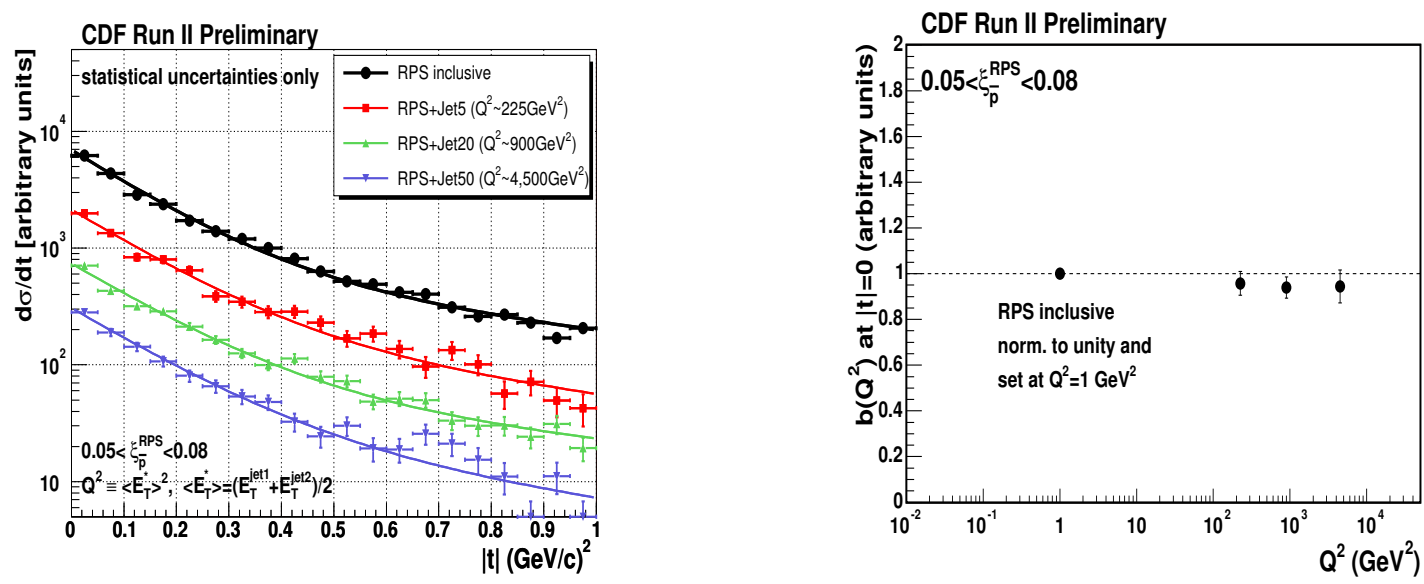

Figure 8: Distribution of $t$ (left) and exponential slope $b$ as measured for different $Q^{2}$ values by the CDF Collaboration.

protons are recorded in all nine spectrometers and a strong effort is ongoing in the alignment and the calibration. With the arrival of new manpower the diffractive analysis program is starting to take off with various analysis topics.

At CDF (K.Goulianos) a similar diffractive program as for Run I is pursued. The already in Run I used Roman pot stations were supplemented in the forward region by the miniplug calorimeters and beam shower counters. For the new preliminary results from Run II a much higher statistics lead to new measurements at very high $Q^{2}$ values up to $10.000 \mathrm{GeV}^{2}$. Only a small $Q^{2}$ dependence is found in the rate of diffractive to non-diffractive dijet production, pointing to a similar evolution of the diffractive parton distributions as seen in the PDF's in the proton.

With the increased statistics also the $t$ dependence could be measured for different $Q^{2}$ values. The $t$ dependences, as seen in the figure 8 , can be described with a double exponential form. Up to values of $|t|=1 \mathrm{GeV}^{2}$ no dips appear and no $Q^{2}$ dependence is visible when rising the $Q^{2}$ from the low values in the soft inclusive data up to the highest achievable values. This goes in-line with the findings at HERA, where the $t$ dependence has been studied for different $Q^{2}$ values by the $\mathrm{H} 1$ and the ZEUS Collaborations. Also in these ep collisions no change with $Q^{2}$ has been observed.

Exclusive production processes are extensively studied with the CDF experiment. As mentioned previously these are excellent testing grounds for the predictions of exclusive production processes at the LHC. CDF reported also on the first observation of exclusive $\gamma \gamma$ production and is now in the process of completing the background estimation.

The new preliminary results on exclusive dijet production are in reasonable good agreement with the predictions of the ExHuME Monte Carlo, also employed for predictions for the LHC. The Tevatron data will help to adjust model parameters for a more precise extrapolation towards LHC energies.

Another hadron-hadron collider presently taking data is RHIC as reported by S.White. Here the main detector component for diffractive measurements is the Zero Degree Calorimeter. The physics menu comprises a broad field of physics topics ranging from total cross section measurements over diffractive vector meson production and deep inelastic $\gamma A$ interactions to leading neu- 
tron production in $p p$ collisions. Having a similar acceptance for leading neutrons as the HERA experiments, the first preliminary spectra resembles the ones measured by ZEUS. The Zero Degree Calorimeter plays a very successful role for RHIC physics and a similar construction has been proposed for a Zero Degree Calorimeter in ATLAS, where it will greatly enlarge the available $\eta$ coverage. These considerations will be discussed in the following paragraph, the next knot in the red line of this summary.

\section{Diffraction at the $\mathrm{LHC}$}

The baseline detector components of the ATLAS and the CMS experiments cover only the central region of the available phase space in pseudo-rapidity $(\eta)$. The forward calorimetry in CMS reaches up to $\eta \approx 5$. Both experiments, ATLAS and CMS have approved projects for additional detector components to complement the $\eta$ coverage. In total five talks (R.Croft, F.Ferro, R.Orava, Chr.Royon, S.White) gave more details on these projects.

Concentrating first on luminosity measurements ATLAS (Chr.Royon) will install a Cerenkov counter (LUCID) at a position of $\eta \approx 6$ and Roman pots at $220 \mathrm{~m}$ away from the interaction point. The goal is to obtain an absolute luminosity by an elastic scattering measurement with the Roman Pots down to the Coulomb region. This measurement is also used for the cross calibration of LUCID, which will serve for a relative luminosity measurement at higher beam luminosities. For later measurements of hard diffraction plans exist in the ATLAS Collaboration to exchange the scintillating fiber detectors in the Roman pots with more radiation hard silicon strip detectors. The ATLAS forward detectors will be complemented by a Zero Degree Calorimeter in a design similar as used by PHENIX at RHIC (S.White).

Since the TOTEM experiment, approved for total and elastic cross section measurements at the LHC, is located in the area of the CMS experiment, the joint coverage in $\eta$ phase space is larger than ever achieved in collider experiments. To the CMS forward calorimeters HF, CASTOR and the Zero Degree Calorimeter, TOTEM adds the tracking stations T1 and T2 and two Roman pot stations at $147 \mathrm{~m}$ and $220 \mathrm{~m}$ on both sides of the experiment. The present status of the TOTEM detector components was reported by F.Ferro.

Just recently CMS and TOTEM documented their common interests for diffractive and forward physics measurements in a joint CMS/TOTEM note [2]. Forward physics at the LHC spans a broad range of high energy physics topics from fundamental properties of QCD to new physics phenomena and the precise determination of the luminosity. A comprehensive overview on the available possibilities was presented by R.Orava. All aspects connected with the gap survival dynamics can be studied in soft and hard diffraction. The structure of the diffractive exchange can be explored in hard diffractive production of jets, $W$-bosons, $J / \Psi$, top and hard photons. Double Pomeron exchange events will serve as a gluon factory. Taging scattered particles in the forward region will allow to investigate low- $x$ phenomena and shed light on the dynamics of the parton evolution and possibly distinguish between regions of DGLAP, BFKL and CCFM dynamics. The areas of parton saturation, diffraction and multi-parton interactions, related by the AGK cutting rules, will be accessible here, too. Other topics like two-photon interactions and peripheral collisions, as studied at RHIC, open another field for physics analyses. Measurements of energy and particle flow and the structure of minimum bias events will lead to new input for cosmic ray data 

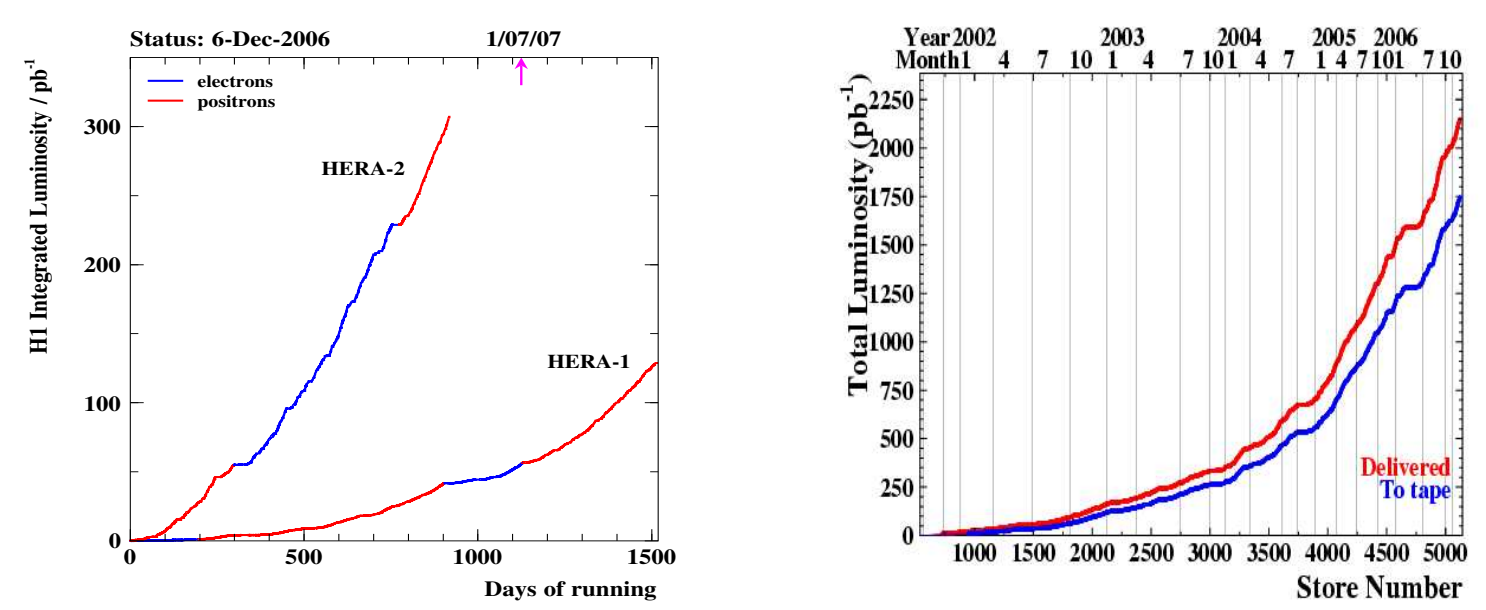

Figure 9: Integrated luminosity delivered by HERA (left) for electron and positron running and integrated luminosity delivered at the Tevatron and collected on tape by CDF.

analyses. The study of QED processes like $p p \rightarrow$ ppee with the forward detectors will be helpful for the accurate determination of the luminosity.

A crucial role for the, also during this workshop, intensively discussed exclusive Higgs production at the LHC, is played by the so-called FP420 project. Here it is planned to install forward proton detectors in the cold region of the beam line at $420 \mathrm{~m}$ away from the interaction point. Interesting facts about experimental aspects were discussed by R.Croft. The FP420 project is a joint effort from members of both, the ATLAS and the CMS Collaborations. The future upgrade after the startup with these forward proton detectors will greatly enhance the diffractive physics opportunities at the LHC. Further expectations for the future are outlined in the following section which builds the last knot of the red line of this summary.

\section{Prospects for the Future}

New and even more precise data are expected from the further operation of HERA II. Only few problems in machine components interrupt occasionally the continuous data taking of the HERA experiments who are on their way to collect a large amount of luminosity as shown in the left picture of figure 9. The switching between electron and positron running is chosen such that approximately equal data samples for each lepton type and polarization will be available in summer 2007, the end of the HERA running. Up to the end of 2006 approximately $500 \mathrm{pb}^{-1}$ will have been delivered in total by the HERA collider. It is planned to have a special HERA running with lower proton energy, $460 \mathrm{GeV}$ instead of the nominal $920 \mathrm{GeV}$, starting in March 2007 . The goal for this special running is to collect in total $10 \mathrm{pb}^{-1}$. With such an amount of data the longitudinal proton structure $F_{L}$ can be determined with enough precision to provide crucial input for the determination of the gluon density at low $x$ and low $Q^{2}$. The experiments H1 and ZEUS are intensively preparing all necessary tools to provide a fast analysis of these precious data. The prospects for the HERMES experiment, using only the lepton beam at HERA, are given by the newly installed proton 
recoil detector. The information provided by this additional detector will open new and promising possibilities especially in DVCS analyses for example.

The right picture in figure 9 the total luminosity delivered by the Tevatron collider. The data on tape are approaching the $2 \mathrm{fb}^{-1}$ mark and the ultimate goal is to collect $4-9 \mathrm{fb}^{-1}$ until the end of operation in 2009.

By then the LHC machine will have started beam operation. Recent plannings foresee the first beams to be injected in November 2007 [3]. They will be brought into collisions with the injection energy of $450 \mathrm{GeV}$. A brief data taking of one or two days will serve to debug the machine and the detectors and will provide first data for alignment and calibration. After a shut down, in which the LHC machine and the experiments will install further components, a first high energy run will start in spring 2008 and continue through the year, accumulating a luminosity of a few $\mathrm{fb}^{-1}$. Running at nominal luminosity will provide approximately $80 \mathrm{fb}^{-1}$ per year. Further luminosity increase without hardware investment is possible by illuminating only two detectors. An even further increase of the luminosity of a factor of two is under study as well as a possible increase of the proton energy.

\section{Summary}

During the workshop all experiments have shown a considerable improvement in the precision of their data and much more new data are available, shedding light on new aspects in diffraction. With the future data taking at the presently running colliders even more precise data and precious special data taking are in view. Of course everybody is looking forward to the first diffractive event produced in $p p$ collisions at the LHC and to pursuing the exciting physics analyses outlined in the bright prospects.

A lot of progress has been achieved so-far, but still a lot remains to be understood. The stimulating atmosphere of the Diffraction 2006 workshop opened the possibility for intensive discussions between the theorists and experimentalists of the various fields. This kind of cross talk is vital to achieve progress in the understanding of diffraction and related forward physics topics. The series of the Diffraction workshops build a solid basis for these discussions and we a looking forward to the next Diffraction workshop planned for 2008.

\section{Acknowledgments}

It is my pleasure to thank the organizers for this enjoyable workshop at such a lovely place. A big THANK YOU goes to all speakers for providing me with their talks well in advance. All talks were so well prepared that it was a lot of fun to cut and paste for the summary.

\section{References}

[1] A.Krisch, proceedings of the EDS’05, Blois, France, 15 - 20 May 2005.

[2] The CMS and TOTEM Collaborations, CMS Note (in preparation), TOTEM Note 06-5, submitted to the LHC Committee CERN/LHCC 2006-039/G-124.

[3] L.Evans, proceedings of the ICHEP'06, Moscow, Russia, 26 Jul - 2 Aug 2006. 